\title{
Characterizing short-wave infrared fluorescence of conventional near- infrared fluorophores
}

Brook K. Byrd

Margaret R. Folaron

Joseph P. Leonor

Rendall R. Strawbridge

$\mathrm{Xu} \mathrm{Cao}$

Petr Bruza

Scott C. Davis 


\title{
Characterizing short-wave infrared fluorescence of conventional near-infrared fluorophores
}

\author{
Brook K. Byrd, ${ }^{a, \dagger}$ Margaret R. Folaron, ${ }^{a, \dagger}$ Joseph P. Leonor, ${ }^{a}$ Rendall R. Strawbridge, ${ }^{a}$ Xu Cao,,${ }^{a, b}$ Petr Bruza, ${ }^{a}$ \\ and Scott C. Davis ${ }^{a, c, *}$ \\ aDartmouth College, Thayer School of Engineering, Hanover, New Hampshire, United States \\ ${ }^{b}$ Xidian University, Engineering Research Center of Molecular and Neuro Imaging, School of Life Science and Technology, Ministry of Education, \\ Xi'an, Shaanxi, China \\ 'Norris Cotton Cancer Center, Dartmouth-Hitchcock Medical Center, Lebanon, New Hampshire, United States
}

\begin{abstract}
The observed behavior of short-wave infrared (SWIR) light in tissue, characterized by relatively low scatter and subdiffuse photon transport, has generated considerable interest for the potential of SWIR imaging to produce high-resolution, subsurface images of fluorescence activity in vivo. These properties have important implications for fluorescence-guided surgery and preclinical biomedical research. Until recently, translational efforts have been impeded by the conventional understanding that fluorescence molecular imaging in the SWIR regime requires custom molecular probes that do not yet have proven safety profiles in humans. However, recent studies have shown that two readily available near-infrared (NIR-I) fluorophores produce measurable SWIR fluorescence, implying that other conventional fluorophores produce detectable fluorescence in the SWIR window. Using SWIR spectroscopy and wide-field SWIR imaging with tissue-simulating phantoms, we characterize and compare the SWIR emission properties of eight commercially available red/NIR-I fluorophores commonly used in preclinical and clinical research, in addition to a SWIR-specific fluorophore. All fluorophores produce measurable fluorescence emission in the SWIR, including shorter wavelength dyes such as Alexa Fluor 633 and methylene blue. This study is the first to report SWIR fluorescence from six of the eight conventional fluorophores and establishes an important comparative reference for developing and evaluating SWIR imaging strategies for biomedical applications. (c) The Authors. Published by SPIE under a Creative Commons Attribution 4.0 Unported License. Distribution or reproduction of this work in whole or in part requires full attribution of the original publication, including its DOI. [DOI: 10.1117/1.JBO.24.3.035004]
\end{abstract}

Keywords: short-wave infrared; near-infrared II window; fluorescence spectroscopy; cancer imaging; medical imaging; fluorescenceguided surgery.

Paper 180553RR received Sep. 19, 2018; accepted for publication Feb. 22, 2019; published online Mar. 8, 2019.

\section{Introduction}

Early reports of fluorescence imaging in the short-wave infrared (SWIR) regime, ${ }^{1-3}$ also known as the near-infrared II (NIR-II) window, have generated substantial interest in the biomedical optics community. Fluorescence imaging in this regime, generally considered to extend from 1000 to $2000 \mathrm{~nm}$, is characterized by reduced photon scatter, minimal tissue autofluorescence, and wavelength-dependent absorption, which can be exploited to optimize image resolution and depth sensitivity. ${ }^{45}$ Broad efforts to leverage these favorable properties for in vivo imaging have facilitated the development of a wide array of SWIRspecific molecular probes used for imaging tumor biomarkers ${ }^{6,7}$ and vascular dynamics ${ }^{2,3,6,8}$ in preclinical models. Although a complete understanding of these properties and their potential implications for biomedical imaging applications has yet to be fully realized, these early studies suggest that SWIR imaging may be highly advantageous for visualizing subsurface structures during fluorescence-guided surgery (FGS), noninvasive monitoring of disease, as well as a wide array of preclinical applications requiring information from molecular reporters.

Until recently, two characteristics of SWIR imaging presented significant barriers to accelerating translational research efforts

*Address all correspondence to Scott C. Davis, E-mail: Scott.C.Davis@ Dartmouth.edu

†The authors contributed equally. in the field. First, the high price point of specialized SWIR cameras remains a significant, though not insurmountable, barrier for many research labs. A more significant barrier arose from the conventional assumption that specialized molecular constructs were required for SWIR fluorescence imaging. These molecular probes can be difficult to obtain, usually manufactured in individual research labs (with a few commercial exceptions), and, importantly, do not yet have pharmacokinetic and safety profiles necessary for mid-to-late-stage translational research. These barriers have generally limited SWIR imaging to animal research in labs with appropriate expertise.

In this context, recent investigations showing robust fluorescence emission signals in the SWIR regime from a handful of conventional NIR-I dyes [indocyanine green (ICG), LI-COR IRDye $800 \mathrm{CW}$, and IR-12N3] represent a major development for the field. ${ }^{9-11}$ ICG is an extensively studied fluorophore approved for human use in several clinical indications, and IRDye $800 \mathrm{CW}$ has been used widely in preclinical models and is under clinical investigation for a variety of indications. ${ }^{12}$ The observation that the tails of the fluorescence emission peaks of these fluorophores are readily detectable well into the SWIR window suggests that other common NIR fluorophores likely produce measurable fluorescence emission in this regime. The availability of SWIR reporters that are accessible, wellcharacterized, and have historic use in biological and human systems could further accelerate development and evaluation of this promising new imaging modality. 
To that end, this study aims to characterize and compare the fluorescence emission profiles in the SWIR regime of eight commercially available red/NIR fluorophores as well as a SWIR-specific fluorophore. Several of these fluorophores are either approved for clinical use or currently in clinical trials, whereas others are commonly used for preclinical in vivo studies. Using a SWIR spectrometer, fluorescence emission spectra for each fluorophore were examined to establish a comparative reference of SWIR fluorescence efficiency for different excitation wavelengths. Additionally, a wide-field imaging system was used to report SWIR fluorescence as a function of concentration in tissue-simulating phantoms. These data will be valuable in guiding the development of SWIR fluorescence imaging strategies for biomedical applications.

\section{Methods and Materials}

The red/NIR-I fluorophores under investigation in this study were selected for their general accessibility and historical use in preclinical and/or clinical studies. ICG (Chem-Impex Int'1. Inc., Wood Dale, Illinois) and methylene blue (Sigma-Aldrich, St Louis, Missouri) are both approved for human use, LI-COR's IRDye 800CW (LI-COR Biosciences, Inc., Lincoln, Nebraska) has undergone clinical investigation for FGS in multiple tumor sites, ${ }^{12}$ and LI-COR's IRDye 680RD and IRDye 700DX, as well as the Alexa Fluor fluorophores (AF 633, AF 647, and AF 750, ThermoFisher Scientific, Waltham, Massachusetts), are used extensively as laboratory standards for in vitro and in vivo imaging. Finally, we included IR-m1050 (Nirmidas Biotech, Inc., Palo Alto, California), one of the few commercially available SWIR-specific fluorophores.

With the exception of ICG, which was dissolved in deionized water, all fluorophores were diluted in phosphate-buffered saline (PBS). The absorbance spectrum for each fluorophore, acquired using a Varian Cary ${ }^{\circledR} 50$ Bio UV-VIS spectrophotometer (Walnut Creek, California), was used to calculate fluorophore dilutions. All fluorophores were diluted to a concentration of $1 \mu \mathrm{M}$ for SWIR fluorescence spectroscopy.

A diagram of the SWIR spectroscopy system used to quantify SWIR fluorescence is shown in Fig. 1(a). The liquid samples were prepared in standard $1-\mathrm{cm}(3 \mathrm{~mL})$ spectroscopy cuvettes and placed in the sample interface, which positioned illumination and collection fibers perpendicular to one another on adjacent cuvette faces. The samples were excited with each of the following five lasers sequentially: $635,730,760,785$, and $670 \mathrm{~nm}$ (World Star Tech, Markham, Ontario, Canada) through a $200-\mu \mathrm{m}$ illumination fiber. All laser power outputs were tuned to $21 \mathrm{~mW} / \mathrm{cm}^{2}$ to ensure fluorescence intensity values were comparable. Emitted light was collected using a spectroscopy fiber bundle terminating in a linear array at the entrance of the spectrograph. The detection system consisted of a Spectra Pro SP2150 (Princeton Instruments, Acton, Massachusetts) spectrograph with a $300-\mathrm{g} / \mathrm{mm}$ grating blazed at $1200 \mathrm{~nm}$, coupled to an InstaSpec IR spectroscopy camera (Newport Corporations, Franklin, Massachusetts). This camera is a $1024 \times 256$ pixel InGaAs transferred-electron electron-bombarded chargedcoupled device cooled to $-50^{\circ} \mathrm{C}$. The detected light passed through an 1100-nm dichroic longpass filter (Thorlabs, Newton, New Jersey) positioned in a filter wheel at the entrance of the spectrometer. All measurements were acquired with a camera gain setting of 800- and 40-ms exposure time. Data from the sensor were binned vertically during acquisition. (a)

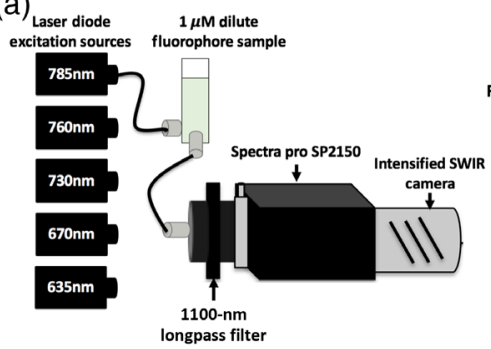

(b)

(c)

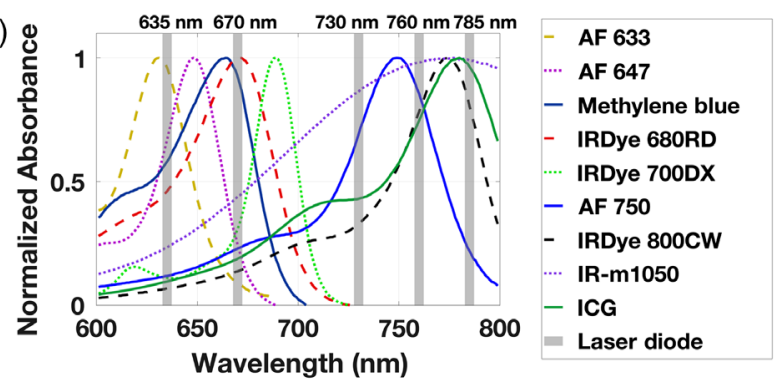

Fig. 1 Experimental configuration for (a) SWIR fluorescence spectroscopy and (b) SWIR phantom imaging. (c) Normalized absorbance spectra of each fluorophore with overlays indicating excitation laser wavelengths used to measure SWIR fluorescence.

For each excitation source-fluorophore combination, 10 SWIR fluorescence spectra frames were acquired sequentially and the median of these 10 frames was computed, a common acquisition/processing strategy for cameras with high dark noise. Background subtraction was performed using dark count spectra without laser illumination. Integrated fluorescence was computed between 1130 and $1200 \mathrm{~nm}$ and normalized to the maximum integrated intensity value measured for all fluorophores (ICG excited at $785 \mathrm{~nm}$ ). Signal-to-noise-ratios (SNRs) were calculated as the mean/(standard deviation) of the integrated intensity values of 10 frames. All measurements were acquired using the same camera settings, laser power, and sample preparation and thus were comparable.

To establish the feasibility of imaging SWIR fluorescence of these fluorophores in more realistic conditions, a concentration dilution study in tissue-simulating phantoms was completed using a wide-field SWIR imaging system. This system, depicted in Fig. 1(b), consists of a chosen laser source fiber coupled to a condenser lens for illumination, and a Nirvana 640 InGaAs camera (Princeton Instruments, Trenton, New Jersey) with an 1100$\mathrm{nm}$ longpass dichroic filter for detection. Tissue-simulating phantoms consisted of 5-mL liquid wells containing $1 \%$ intralipid and $1 \%$ whole blood in PBS to approximate photon scatter and absorption of tissue, respectively, and the dye of interest. The concentration of each dye was varied from $5 \mu \mathrm{M}$ to $19 \mathrm{nM}$ in 50\% serial dilutions. The excitation lasers used for each phantom were as follows: $635 \mathrm{~nm}$ for AF 633 and AF 647; $670 \mathrm{~nm}$ for methylene blue, IRDye 680RD, and IRDye 700DX; $760 \mathrm{~nm}$ for IRDye 800CW, AF 750, IR-m1050, and ICG. The excitation power densities measured at the sample surface ranged between 25 and $74 \mathrm{~mW} / \mathrm{cm}^{2}$ depending on laser source. Five 200-ms images were acquired for each dye concentration. Images were scaled to the excitation intensity and baselined using the intensities measured from phantoms containing no fluorophore for each laser, though were not adjusted based on dye absorbance. Mean values of a region of interest in each phantom were used to report the measured intensity as a function of dye concentration, 
and SNR was calculated as the mean/(standard deviation) of the five frames. For each dye, linear fits and Pearson's coefficient were determined using measurements with $\mathrm{SNR}>5$.

\section{Results}

The absorbance spectra for all fluorophores under investigation are plotted with the overlapping laser diode excitation wavelengths in Fig. 1(c). Representative SWIR fluorescence spectra and corresponding integrated signal values are shown in Figs. 2(a)-2(f), respectively. The characteristic longpass filter cut-on is observable at $1100 \mathrm{~nm}$ in the spectral plots, and the spectral features of these measurements are consistent with the tails of the fluorophores' emission peaks. The complete set of integrated signal values was normalized to the highest signal value (ICG excited at $785 \mathrm{~nm}$ ) and plotted in Fig. 2(g) for every excitation source-fluorophore pairing. For these measurements, the maximum SNR for each dye was as follows: $\mathrm{AF} 633=5, \mathrm{AF}$ $647=10$, methylene blue $=6$, IRDye $680 \mathrm{RD}=22$, IRDye $700 \mathrm{DX}=4$, AF $750=37$, IRDye $800 \mathrm{CW}=49$, IR-m1050 = 38 , and $\mathrm{ICG}=38$.
Inspection of Fig. 2 indicates that in spectroscopy mode (dilute, nonturbid solutions), all fluorophores under investigation generated measurable fluorescence in the SWIR window under proper illumination conditions, and the intensity of the emission matched expectations based on the characteristic absorbance spectrum of each fluorophore. As expected, fluorophores with absorbance and emission peaks at shorter wavelengths-AF 633, AF 647, methylene blue, IRDye 680RD, and IRDye 700DX - generated the lowest signal intensities in the SWIR window, and the highest fluorescence intensities were observed for the longer wavelength fluorophores. The strongest fluorescence emission signal of all fluorophores was observed from ICG when illuminated with 785-nm excitation light, though IRDye $800 \mathrm{CW}$ and IR-m1050 also showed robust fluorescence emission. These results are consistent with the absorbance spectra for each fluorophore.

Images and quantitative analysis of the tissue-simulating phantoms, acquired with the wide-filed imaging system as shown in Fig. 1(c), are presented in Fig. 3. Figure 3(a) shows representative images of dye dilutions for AF 633, IRDye
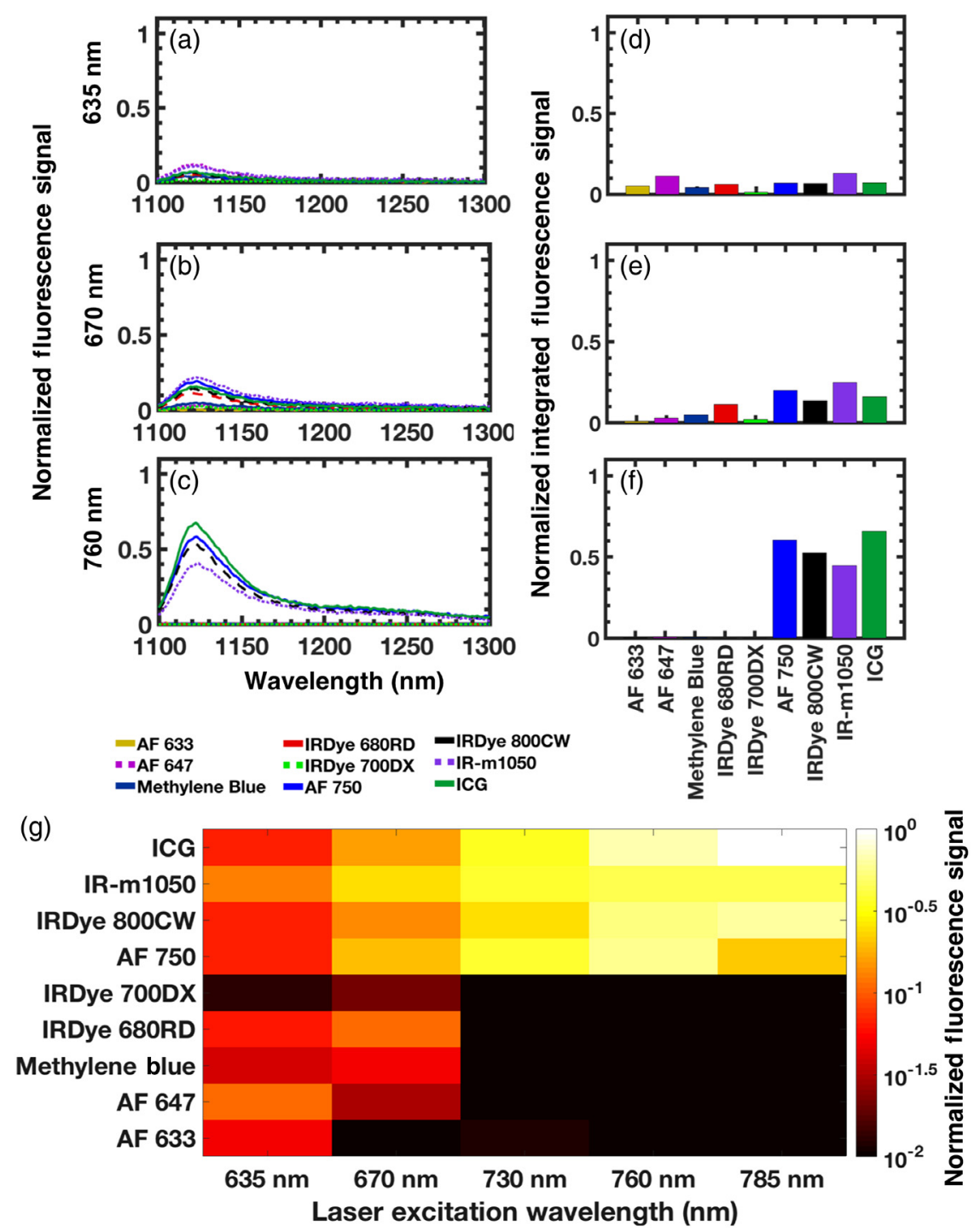

Fig. 2 (a)-(c) Normalized fluorescence emission spectra and (d)-(f) corresponding integrated fluorescence signals for three excitation sources. (g) Normalized integrated SWIR fluorescence for each fluorophore/excitation source investigated. 
(a)

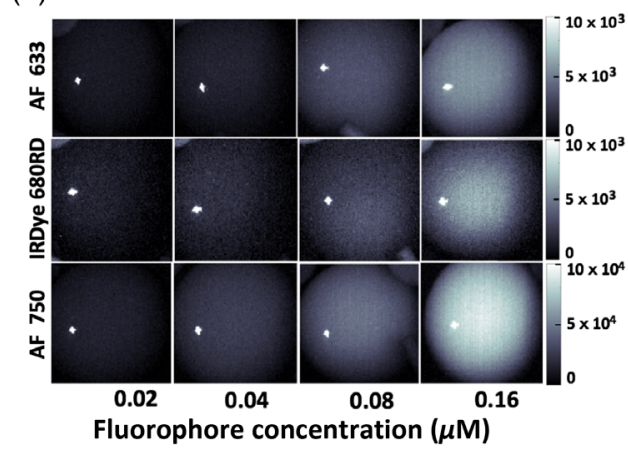

(c)

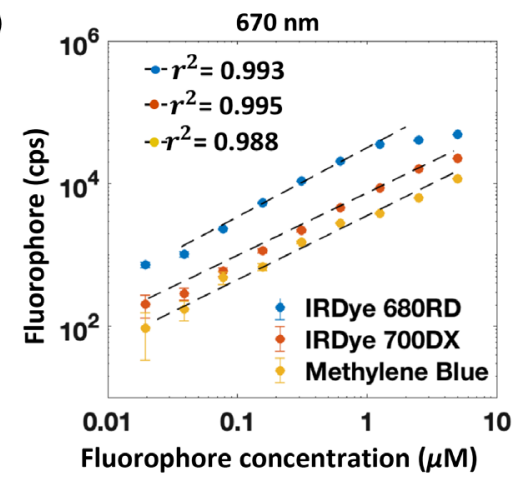

(b)
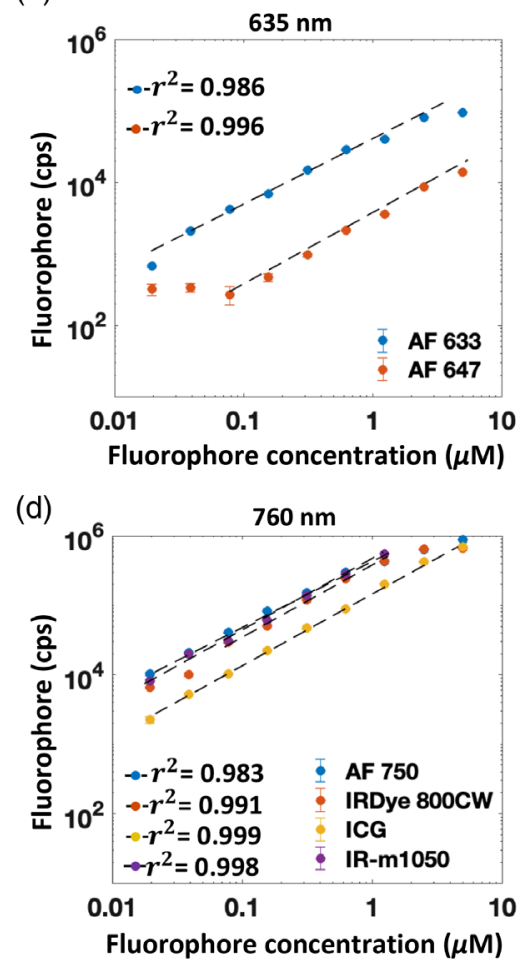

Fig. 3 (a) Representative images of SWIR fluorescence in tissue-simulating phantoms containing AF 633, IRDye 680RD, and AF 750. Each image is the median of five acquisitions. (b)-(d) Fluorescence intensities of phantom images as a function of fluorophore concentration (error bars are standard deviation) with linear fits and Pearson's coefficients.

680RD, and AF 750 over a selected range of concentrations. Qualitatively, these images show responses to changing dye concentration indicative of sensitivity to the dyes. A quantitative analysis of all dyes are presented in Figs. 3(b)-3(d) and confirm the qualitative observations. These panels show the mean fluorescence intensity values of all dyes as a function of dye concentration for 635-, 670-, and 760-nm excitation sources, respectively, with linear fits to the data. For reference, the baseline values for phantoms without fluorophores were 1560, 820, and 6030 counts/s for the 635-, 670-, and 760-nm lasers, respectively (these values were subtracted from the data shown in Fig. 3 before plotting). The SNRs for all dyes at $156 \mathrm{nM}$ were as follows: $\mathrm{AF} 633=150, \mathrm{AF} 647=8$, methylene blue $=13$, IRDye $680 \mathrm{RD}=22$, IRDye 700DX $=16, \mathrm{AF} 750=281$, IRDye $800 \mathrm{CW}=150$, IR-m1050 $=221$, and $\mathrm{ICG}=119$.

Examination of Fig. 3 confirms that SWIR fluorescence from all dyes studied is readily detectable in imaging mode over a large range of biologically relevant concentrations using a simple imaging system. With the exception of AF647, for which the minimum detectable concentration was between 100 and $200 \mathrm{nM}$, all dyes showed a linear response down to the lowest concentration measured $(20 \mathrm{nM})$.

\section{Discussion}

This study is the first to report SWIR fluorescence from AF 633, AF 647, methylene blue, IRDye 680RD, IRDye 700DX, and AF 750, and provides a comprehensive, quantitative comparison of SWIR fluorescence efficiency among a catalog of commonly used fluorophores. SWIR fluorescence was readily detectable in nonturbid samples measured using spectroscopy and in turbid tissue-simulating phantoms imaged with an epi-illumination
SWIR imaging system. The results from the imaging study are particularly striking and showed a robust linear response down to the lowest concentrations imaged $(20 \mathrm{nM})$ for nearly all fluorophores. Although many of these dyes will likely be detectable at even lower concentrations, this degree of sensitivity is already well within the range commonly encountered during in vivo molecular imaging.

The relatively robust SWIR fluorescence for the shorter wavelength fluorophores (AF 633, AF 647, methylene blue, IRDye 680RD, and IRDye 700DX) is particularly noteworthy. Although using these shorter wavelength fluorophores to image in the SWIR window will have inherent limitations, namely, lower fluorescence intensity and shorter wavelength excitation sources that may not penetrate as deeply in tissue, there are likely specific instances for which these fluorophores offer unique benefits. For example, methylene blue is commonly used for sentinel lymph node mapping during cancer resection and has been under clinical investigation for other indications. ${ }^{13}$ Thus, clinical evaluation of SWIR fluorescence for this indication could be undertaken without the need for an investigational drug designation.

The fluorescence intensity heat map shown in Fig. 2 can be used as a reference for comparing SWIR fluorescence efficiency between the fluorophores tested; however, it should be noted that some dyes were not excited at their optimal wavelength. This can be accommodated by scaling the results in the figure to the normalized absorbance spectra of each dye.

The general trends observed in the spectroscopy measurements and the imaging experiments were comparable, although the relative intensities between some dyes changed. This is most likely due to the well-known effects of changing chemical 
environments. For example, the reversal of relative intensities of AF 633 and AF 647 between spectroscopy and phantom measurements is likely due to the fluorophores' well-documented behavior in lipid-based solutions. ${ }^{14}$ This represents the most dramatic change observed between two dyes, and discrepancies between the two systems for other dyes were modest.

To our knowledge, the results herein are the first to report and compare methodically the SWIR fluorescence from a large catalog of red/NIR fluorophores. The observation that all fluorophores investigated produced measurable fluorescence expands the catalog of fluorophores that can be used for translational research of SWIR molecular imaging. Many of these fluorophores are used in clinical practice, are under clinical investigation, or are regularly used in preclinical animal research. Thus, the findings presented herein offer a valuable resource to accelerate the development and evaluation of this promising imaging modality.

\section{Disclosures}

The authors have no conflicts of interest to disclose.

\section{Acknowledgments}

The authors thank the Physical Sciences Inc. for access to the InstaSpec camera. This work was funded by the National Institutes of Health under Grant Nos. R01CA184354 and R01CA188491 (SC Davis).

\section{References}

1. K. Welsher et al., "A route to brightly fluorescent carbon nanotubes for near-infrared imaging in mice," Nat. Nanotechnol. 4(11), 773-780 (2009).
2. G. Hong et al., "Multifunctional in vivo vascular imaging using nearinfrared II fluorescence," Nat. Med. 18(12), 1841-1846 (2012).

3. D. J. Naczynski et al., "Rare-earth-doped biological composites as in vivo shortwave infrared reporters," Nat. Commun. 4, 2199 (2013).

4. R. H. Wilson et al., "Review of short-wave infrared spectroscopy and imaging methods for biological tissue characterization," J. Biomed. Opt. 20(3), 030901 (2015).

5. J. A. Carr et al., "Absorption by water increases fluorescence image contrast of biological tissue in the shortwave infrared," Proc. Natl. Acad. Sci. U.S.A. 115(37), 9080-9085 (2018).

6. Z. Xue, S. Zeng, and J. Hao, "Non-invasive through-skull brain vascular imaging and small tumor diagnosis based on NIR-II emissive lanthanide nanoprobes beyond 1500 nm," Biomaterials 171, 153-163 (2018).

7. Z. Tao et al., "Early tumor detection afforded by in vivo imaging of nearinfrared II fluorescence," Biomaterials 134, 202-215 (2017).

8. O. T. Bruns et al., "Next-generation in vivo optical imaging with shortwave infrared quantum dots," Nat. Biomed. Eng. 1(4), 0056 (2017).

9. J. A. Carr et al., "Shortwave infrared fluorescence imaging with the clinically approved near-infrared dye indocyanine green," Proc. Natl. Acad. Sci. U.S.A. 115(17), 4465-4470 (2018).

10. Z. Starosolski et al., "Indocyanine green fluorescence in second nearinfrared (NIR-II) window," PLoS One 12(11), e0187563 (2017).

11. S. Zhu et al., "Repurposing cyanine NIR-I dyes accelerates clinical translation of near-infrared-II (NIR-II) bioimaging," Adv. Mater. 30(34), 1802546 (2018)

12. E. L. Rosenthal et al., "Safety and tumor specificity of cetuximabIRDye800 for surgical navigation in head and neck cancer," Clin. Cancer Res. 21(16), 3658-3666 (2015).

13. J. R. van der Vorst et al., "Intraoperative near-infrared fluorescence imaging of parathyroid adenomas with use of low-dose methylene blue," Head Neck 36(6), 853-858 (2014).

14. L. D. Hughes, R. J. Rawle, and S. G. Boxer, "Choose your label wisely: water-soluble fluorophores often interact with lipid bilayers," PLoS One 9(2), e87649 (2014).

Biographies of the authors are not available. 